Original Paper http://ajol.info/index.php/ijbcs http://indexmedicus.afro.who.int

\title{
Caractérisations morphologique et génétique d'espèces d'aleurodes prélevées dans les cultures de tomate atteinte de la virose du $T Y L C$, dans la zone des Niayes au Sénégal
}

\author{
Mouhameth CAMARA ${ }^{1}$, Serigne Modou SARR ${ }^{1 *}$, Ibrahima SARR $^{2}$ et \\ Kandioura NOBA ${ }^{3}$ \\ ${ }^{I}$ Université de Thiès, Institut Supérieur de Formation Agricole et Rurale (ISFAR) ex ENCR de Bambey, BP 54 \\ Bambey, Sénégal. \\ ${ }^{2}$ Centre National de Recherches Agricoles (CNRA)/de Bambey, BP 53 Bambey, Sénégal. \\ ${ }^{3}$ Université Cheikh Anta Diop de Dakar (UCAD), Département de Biologie Végétale, Dakar, Sénégal. \\ "Auteur correspondant; E-mail: sarmodou@yahoo.fr ; Tél: (00221) 774358017
}

\section{RESUME}

Les aleurodes sont vecteurs de plusieurs virus dont celui du jaunissement et de l'enroulement des feuilles de tomate (Solanum lycopersicum L.) appelé Tomato Yellow Leaf Curl Virus (TYLCV). L'objectif de ce travail était d'identifier les différentes espèces d'aleurodes présentes dans les cultures de tomate de la zone des Niayes en rapport avec le TYLC. Des puparia et des adultes ont été récoltés sur les feuilles de la variété "Xina », atteintes du TYLC pour des caractérisations morphologique et génétique. La méthode d'analyse du Ministère français de l'Agriculture a été utilisée pour leur montage et leur identification. Pour six spécimens, l'échantillon le plus proche sur Q-bank et Genbank était un aleurode identifié comme Bemisia tabaci avec une similarité de 75 à $98 \%$. Pour les deux autres, l'aleurode le plus proche dans Q-bank était $B$. afer avec une similarité de 77 à $78 \%$, et dans Genbank B. tabaci avec 77\%. Cinq autres ont été identifiés comme Aleurodicus. Leurs séquences du gène $\mathrm{CO} 1$ sont identiques. Les 11 spécimens identifiés dans Q-Bank comme Aleurodicus dispersus sont identiques à $100 \%$ à aux spécimens du Sénégal. Au total, l'étude a mis en évidence deux espèces différentes de Bemisia (B. tabaci et B. afer) et Aleurodicus dispersus.

(C) 2016 International Formulae Group. All rights reserved.

Mots clés : TYLCV, Bemisia spp, virus, Puparium, séquences.

\section{Morphological and genetic characterizations of whiteflies species sampled from tomato infected by tomato yellow leaf virus disease in Niayes area of Senegal}

\begin{abstract}
Whiteflies are vectors of many viral diseases including tomato (Solanum lycopersicum L.) curling i.e. Tomato Yellow Leaf Curl Virus (TYLCV). This work aimed at identifying different whitefly species on tomatoes in relation to the TYLCV in Niayes areas Puparia and adults were collected from "Xina" tomato variety leaves showing TYLC symptoms for both morphological and genetic characterizations. The French Ministry of agriculture analysis method was used for their preparation and identification. For six specimens,
\end{abstract}


the most similar sample on Q-bank and Genbank was identified as Bemisia tabaci with a similarity ranging between 75 and 98\%. Two others were closest to Bemisia afer on Q-bank with a similarity of 77 to $78 \%$, and Bemisia tabaci on Genbank with a similarity of $77 \%$. Five others were identified as Aleurodicus. Their CO1 gene sequences were similar. The 11 specimens identified as Aleurodicus dispersus with a $100 \%$ similarity to specimens from Senegal. Overall, this study came out with two different species of Bemisia (B. tabaci et B. afer) and Aleurodicus dispersus.

(C) 2016 International Formulae Group. All rights reserved.

Keywords: TYLCV, Bemisia spp, virus, Puparium, sequences.

\section{INTRODUCTION}

Au Sénégal, la maladie du TYLC est apparue en 1980 dans les régions de Kaolack et Ziguinchor (Ciss, 2004). L'apparition de cette maladie est probablement due à la proximité de la culture du coton qui héberge l'insecte vecteur, Bemisia tabaci. Aujourd'hui, elle a gagné tout le territoire national et constitue une menace sérieuse pour la production de la tomate dans le pays, voire dans la sous-région (Camara, 2012).

Selon Ciss (2004), au Sénégal, Bemisia tabaci (Gennadius, 1889) est un ravageur des cultures. Les plus affectées sont : le manioc, le coton, la tomate et autres cultures maraîchères.

La mouche blanche, Bemisia tabaci, a été décrite en 1889 en Grèce sur le tabac par Gennadius sous le nom d'Aleurodes tabaci. Elle appartient à l'ordre des Hémiptères et à la famille des Aleyrodidae; on l'appelle aussi «mouche blanche du coton» ou «mouche blanche de tabac». C'est un aleurode très polyphage signalé aujourd'hui sur plus de 506 plantes appartenant à 74 familles (Diatta, 1999). En Afrique, plus de 150 plantes appartenant à 30 familles victimes des piqûres de ce ravageur, parmi lesquelles des Légumineuses (arachide, haricot), des Malvacées (gombo), des Solanacées (tomate, pomme de terre, aubergine), des Cucurbitacées (melon, pastèque, concombre, courgette), des Crucifères (chou, radis, navet), des Euphorbiacées (manioc), des Agrumes et diverses plantes ornementales ont été recensées (Delatre, 1973).

Cet insecte entraîne des dégâts trophiques directs à cause du miellat qu'il excrète et véhicule de nombreux virus sur les cultures dont celui de la virose de la tomate, le TYLC. Ce virus affecte la fonction photosynthétique des plantes et la qualité des récoltes (Brown, 1994; Traboulsi, 1994 ; Schulten, 1997 ; Bouchelta et al., 2005).

B. tabaci a été signalé par Messiaen et al. (1991) comme étant un agent important de la transmission des virus de la mosaïque du coton en Afrique Occidentale et en Amérique Centrale, des virus « leaf curl» sur le tabac, la tomate et le haricot (Phaseolus spp) et du virus de la mosaïque africaine du manioc en Afrique Tropicale.

Une plasticité écologique impressionnante complique le statut taxonomique de Bemisia tabaci. Cette variabilité a conduit à la mise en évidence de nombreux biotypes (Reynaud, 2000). Le biotype B, initialement identifié dans l'Arizona (USA), est considéré comme le biotype responsable de la majorité de dégâts notés dans le monde, il est probablement originaire du Moyen-Orient (Frohlich et al., 1999). Aucun élément morphologique ne permet au champ comme au laboratoire de différencier le biotype B (parfois appelé $B$. argentifolii) des autres biotypes. Ce biotype se caractérise par son extrême polyphagie (plus de 600 plantes hôtes) et sa facilité à transmettre des virus (Secker et al., 1998). Plus récemment des auteurs (De Barro et al., 2011) considèrent que $B$. tabaci est un complexe contenant au moins 11 groupes bien définis et 24 espèces morphologiquement indiscernables.

Dans le but de connaître les différentes espèces d'aleurodes présentes dans les cultures de tomate de la région de Dakar, des échantillons ont été prélevés pour les 
caractérisations morphologique et génétique. En effet, ces caractérisations sont importantes car peu d'informations existaient sur le virus et son vecteur au niveau de la zone d'étude. Elles sont également importantes pour la compréhension des épidémies virales et pour l'élaboration de stratégies de lutte adéquate. Ainsi, un bégomovirus apparemment proche du ToLCMLV a été identifié et partiellement caractérisé à partir d'un échantillon de feuilles de tomate présentant des symptômes caractéristiques d'une maladie à bégomovirus, et deux espèces différentes de Bemisia sur les tomates cultivées dans le site : $B$. tabaci avec certitude et une espèce probablement proche de B. afer. Concernant cette deuxième espèce, elle n'aurait pas été encore signalée au Sénégal.

\section{MATÉRIEL ET MÉTHODES}

Le matériel biologique est constitué d'adultes et de puparia d'aleurodes et la méthode utilisée s'articule en quatre parties :

\section{Echantillonnage des puparia et adultes d'aleurodes}

Les échantillons ont été récoltés au hasard, sur les feuilles d'une variété de tomate «Xina » (Solanum lycopersicum L.), présentant les symptômes viraux du Tomato Yellow Leaf Curl (TYLC). Ils ont été prélevés au niveau de parcelles expérimentales de tomate de Sangalkam (Latitude $14^{\circ} 46 \mathrm{~N}$, Longitude $-17^{\circ} 13 \mathrm{~W}$, Altitude $19 \mathrm{~m}$ ), dans la région de Dakar, en janvier 2011. Ce qui correspond à la période de forte infestation des parcelles de tomate par les aleurodes dont le pic se situe entre décembre et mars (Ciss, 2004).

\section{Préparation et identification morphologiques des puparia}

Pour caractériser une espèce d'aleurode il faut recourir à l'examen du puparium, le dernier stade larvaire (Martin, 2003). Dans le cadre de cette étude, nous n'avons pu prélever que peu de puparia et nous avons également échantillonné des adultes.
La méthode d'identification morphologique utilisée est la méthode officielle d'analyse des aleurodes d'intérêt agronomique du Ministère français de l'alimentation, de l'agriculture et de la pêche (www.agriculture.gouv.fr, mai 2011). Cette méthode permet l'identification de Bemisia tabaci par observation au microscope photonique des caractères morphologiques des puparia montés entre lame et lamelle. Pour la préparation et le montage des spécimens, les étapes de la partie A de la méthode d'analyse officielle ont été utilisées, l'identification a été faite par la partie B. Cette méthode permet de confirmer l'appartenance des spécimens au complexe $B$. tabaci mais pas d'identifier précisément les différents biotypes. Les puparia ont été éclaircis à la potasse, puis rincés à l'eau distillée et déshydratés par deux bains d'alcool successifs respectivement à 70 et $95 \%$. Un dernier bain dans l'essence de lavande est réalisé avant montage dans le baume du Canada.

\section{Préparation et identification morphologiques des adultes}

Les adultes ont été montés entre lame et lamelle en acide lactique à chaud. L'identification a été faite en utilisant la clé de Guimaraës (1996) qui permet de caractériser 6 espèces d'intérêt agronomique dont $B$. tabaci et les critères supplémentaires proposés par Streito (2005). Les spécimens ont été comparés aux descriptions faites par Malumphy et al. (2009) et aux spécimens de référence de la collection du LSV. Le stade adulte de nombreuses espèces d'aleurodes n'ayant jamais été décrit, malgré tout, l'identification des adultes n'est pas certaine. L'identification moléculaire par barcoding a été faite parallèlement.

\section{Identification moléculaire des adultes}

Les adultes non montés entre lame et lamelle ont été séquencés au CBGP (Centre de Biologie pour la Gestion des Populations) et les résultats obtenus ont été comparés avec les données Q-bank puis avec celles d'Arthropode de l'INRA. En complément, une 
assignation a été faite dans la base de données GenBank NCBI à l'aide du programme « Somewhat similar sequences (Blastn)».

\section{RÉSULTATS}

Les aleurodes récoltés et observés aux trois dates sont consignés dans le Tableau 1. Les deux puparia analysés appartiennent sans ambiguïté au complexe Bemisia tabaci. En effet, ils présentent, au niveau de l'orifice vasiforme, un sillon caudal court et des soies fortes et bien développées (Photo A).

L'identification des adultes a par contre mis en évidence deux espèces différentes : $B$. tabaci, et une autre espèce de Bemisia, indiscernable morphologiquement de $B$. tabaci mais nettement différente génétiquement.

Une analyse moléculaire de 9 adultes de Bemisia présents dans les échantillons a été effectuée et 8 séquences du gène $\mathrm{CO} 1$ ont été obtenues (Figure 1). De même 5 adultes d'Aleurodicus ont été séquencés (Figure 2).

Il n'a été pas possible de différencier morphologiquement les adultes, notamment de Bemisia afer et de B. tabaci. Ainsi, Bemisia $s p$. a été retenu pour les adultes. Deux pupariums avaient été prélevés ultérieurement et correspondaient morphologiquement à Bemisia tabaci au sens large.

Les 8 séquences de $\mathrm{CO} 1$ n'étaient pas identiques et aucun spécimen des bases Qbank ou Genbank ne correspondait à ces séquences. Pour six spécimens, l'échantillon le plus proche sur Q-bank et Genbank était un aleurode identifié comme B. tabaci avec une similarité variant de 75 à $98 \%$. Avec les deux spécimens (JSTR00056_0101 et 0104), l'aleurode le plus proche dans Qbank était Bemisia afer avec une similarité de 77 à $78 \%$ et dans Genbank Bemisia tabaci avec une similarité de $77 \%$. Dans tous les cas, il a été impossible de conclure à une identification spécifique.

Les échantillons JSTR00056_0201 à 0205 ont été identifiés morphologiquement comme appartenant au genre Aleurodicus et ne différaient en rien des adultes $A$. dispersus présents dans la collection du Laboratoire de la Santé des Végétaux (LSV).

Cinq séquences du gène $\mathrm{CO} 1$ correspondant à 5 adultes de l'échantillon ont été obtenues et s'étaient révélées identiques. La base de données Q-Bank contient 11 spécimens identifiés comme A. dispersus dont la séquence $\mathrm{CO} 1$ était conforme aux spécimens du Sénégal (100\% de recouvrement et $100 \%$ de similarité). L'interrogation de la base Genbank donne deux séquences identiques à $100 \%$ avec les mêmes échantillons.

Compte tenu des analyses morphologiques et des résultats moléculaires concordants, il a été possible de confirmer l'identité spécifique des spécimens d'Aleurodicus: A. dispersus Russell, 1965.

Tableau 1 : Résultats d'observation d'aleurodes sur feuilles de tomates atteintes de TYLC.

\begin{tabular}{ccccc}
\hline Dates d'observation & Tubes & $\begin{array}{c}\text { Nombre de folioles } \\
\text { observées }\end{array}$ & $\begin{array}{c}\text { Organismes } \\
\text { détectés }\end{array}$ & $\begin{array}{c}\text { Stade détecté et } \\
\text { nombre }\end{array}$ \\
\hline Première date & 1 & 2 & Bemisia $s p$ & 10 adultes \\
& 2 & 3 & Bemisia $s p$ & 11 adultes \\
Deuxième date & 3 & 3 & Bemisia $s p$ & 15 adultes \\
(1 semaine après) & 5 & 5 & Bemisia $s p$ & 60 adultes \\
Troisième date & 1 & 2 & Aleurodicus sp & 6 adultes \\
(1 semaine après) & & 3 & & 2 puparia \\
\hline
\end{tabular}




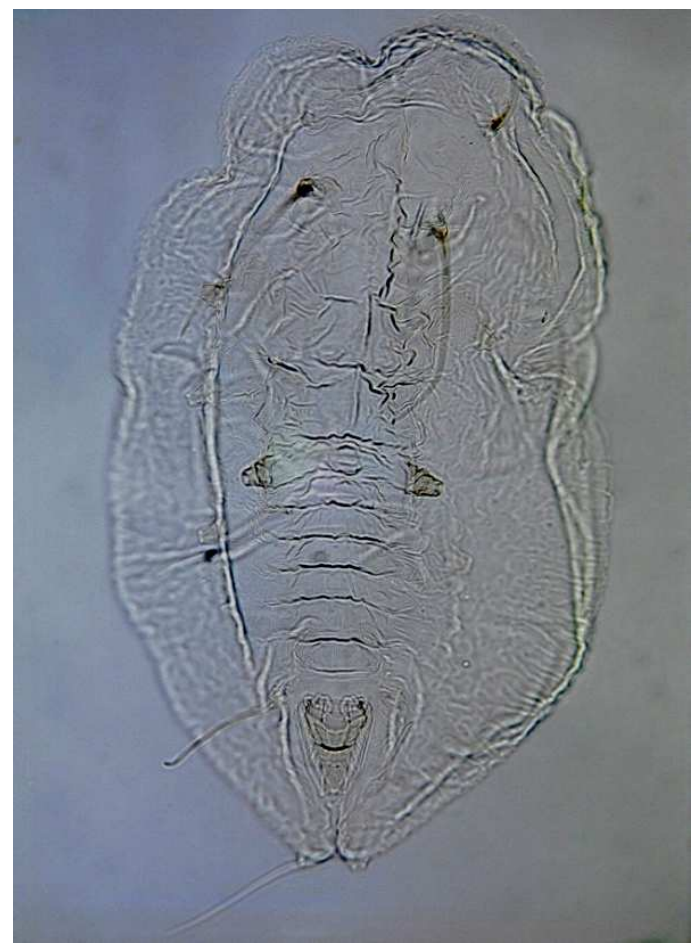

Photo A : Puparium de Bemisia tabaci G x 100.

JSTR00055_0101

échec PCR

JSTR00055_0102

TGTTTTGTATTTTATTTTTGGTGTTTGAAGAGGATTAATTGGAACTTCTTTTAGTATAAT TATTCGCTCTGAGCTTATAAATGTTGGGTCTTTTATATCAAATGAGCATCTATATAATGT TGTTGTAACTTCTCATGCTTTTATTATGATTTTTTTTATAACAATACCACTAGTTATCGGT GGTTTTGGTAATTGGCTTGTTCCTCTGATAATTGGTGCTCCTGACATAGCTTTTCCTCGT ATAAATAACTTGAGTTTTTGACTTTTAGTTCCTTCATTAATTTTTATGTTGGTAAGAATG ATTATTAGGACAGGAGCTGGCACTGGCTGAACTGTTTATCCTCCTCTGTCTTTAAGGTT AACTCATAGAGGCTTATCAGTTGATTTACTAATTTTTTCTTTGCATATTGCAGGTGTTTC ATCAATTCTAGGTTCAGTCAACTTTATTGTTACTATTTTTAATATACGGGTTCTTGGCAT AAACTTTGAATATCTGAGGCTGTTTGTTTGATCAGTATTAATTACAGTATTTTTATTACT GATTTCTCTTCCTGTCCTTGCAGGGGCGATTACAATATTGTTAATAGATCGAAATTTTAA TAGATCTTTTTATGATCCTTTAGGAGGAGGTGATCCTATTTTATATCAGCACTTGTTC JSTR00055_0103

TGTTTTATATTTTATCTTTGGTGTCTGGAGTGGTTTAATTGGGACTTCTTTTAGTATTATT ATTCGTTCTGAACTTATAAATGTAGGACCATTTTTGGCAAATGACCATTTGTATAATGTT GTAGTTACTTCTCATGCGTTTATTATAATCTTTTTTATAACGATACCTTTAGTTATCGGT GGATTTGGGAATTGATTAATTCCTCTAATAATTGGTGCTCCTGACATAGCTTTTCCTCGT ATAAATAATTTAAGGTTTTGACTTCTTGTTCCTTCTCTAATTTTTATATTAATGAGAATA GTTATTAGTCTAGGATCAGGTACTGGTTGGACGGTGTACCCTCCCCTGTCAATAAGACT GACTCACAGGGGGCTTTCGGTTGATCTATTAATTTTTTCTTTACATATTGCAGGTGTGTC TTCAATTTTAGGGTCAGTAAATTTTATTGTTACCATTTTTAATATACGTGTTGTTGGTAT 
AAATTTTGAGTATCTAAGTTTATTTGTGTGATCTGTATTAATTACGGTGTTTTTATTACT TATTTCTCTTCCTGTTCTCGCAGGAGCGATCACTATGTTACTGATGGACCGAAATTTTAA TAGATCTTTCTATGATCCTTTAGGAGGAGGAGATCCTATTTTGTATCAACACTTATTT JSTR00055_0104 TGTTTTATATTTTATTTTTGGTGTTTGAAGAGGATTAATTGGAACTTCTTTTAGTATAAT TATTCGCTCTGAGCTTATAAATGTTGGGTCTTTTATATCAAATGAACATTTATATAATGT TGTTGTAACTTCTCATGCTTTTATTATGATTTTTTTTATAACAATGCCACTAGTTATCGGT GGTTTTGGTAATTGGCTTATTCCTCTGATAATTGGTGCTCCTGACATAGCTTTTCCTCGT ATGAATAACTTGAGGTTCTGACTTTTAGTTCCTTCATTAATTTTTATGTTGGTAAGAATG ATTATTAGGACAGGAGCTGGTACTGGCTGAACTGTTTATCCTCCCCTGTCTTTAAGATT AACTCATAGAGGTTTATCAGTTGATTTATTAATTTTTTCTTTGCACATTGCAGGTGTTTC ATCAATTCTAGGTTCAGTCAACTTTATTGTCACTATTTTTAATATACGGACTCTTGGCAT AAACTTTGAATATCTGAGGCTATTTGTTTGATCAGTATTAATTACAGTATTTTTATTACT GATTTCTCTTCCTGTTCTCGCAGGGGCGATTACAATATTGTTAATAGATCGAAATTTTAA TAGATCTTTTTATGATCCTCTAGGAGGAGGTGATCCCATTTTATATCAGCACTTGTTC

JSTR00056_0101

TATATTATACTTTATTTTTGGTATTTGGAGTGGTCTCATCGGAACTTCATTCAGGATGTT AATTCGGTCTGAATTGATAAGGGTGGGCTCTTTTCTGATAAATGATCAATTATATAATG TTGTTGTCACTTCACATGCGTTCATTATGATTTTTTTTATAACCATACCTTTAGTAATTG GTGGATTCGGGAACTGGCTAGTACCTCTTATAATCGGTGCGCCCGATATAGCGTTTCCT CGAATAAATAATCTTAGGTTTTGACTATTAATCCCCTCCTTAATATTTATAATAACTAGA ATGTTGGTGGGAAGCGGAGCTGGGACGGGGTGAACCGTTTATCCGCCCCTCTCATTGG GGTTAGCTCATAGTGGGTCTTCAGTTGATCTGCTGATCCTGGCCTTACATGTCGCAGGC ATCTCATCTATCTTAGGGTCTGTTAATTTTATTGTTACTATTTTCAACATGCGCGTACTG GGTATAAGATTTGAGTATGTTAGCTTGTTTGTTTGGTCTGTTTTAATCACTGTGTTTTTG TTGCTTATCTCTCTGCCCGTTTTGGCGGGGGCAATTACTATGCTACTGATGGATCGAAA TTTCAATAGGTCATTTTATGACCCCTTGGGGGGAGGGGATCCCGTTCTATACCAACATC TATTT JSTR00056_0102

TTTATATAATTATCCGTTCTGAACTTATAGAGGTGGGGTCTTTTTTAGCAAATGAACATT TATATAATGTTGTTGTAACTTCCCATGCTTTCATTATAATTTTTTTTATAACAATACCGTT AGTTATTGGTGGGTTTGGAAATTGACTTGTTCCTTTAATGATTGGTTCTCCTGACATGGC TTTTCCTCGTATAAACAATTTAAGGTTTTGACTTCTTGTTCCTTCATTGATCTTTATACTA TTTAGTATACTTGTTAGTGTGGGTGCTGGAACCGGTTGGACCGTTTATCCNCCTCTGTCT TTAAGGTTGACTCATAGTGGTTTATCAGTTGATTTATTAATTTTTTCTTTACATATTGCG GGCGTGTTTTCAATTTTAGGTTCAGTTAATTTTATTGTCACTATTTTTAATATACGGGTT CTTGGTATAAATTTTGAGTATTTAAGATTGTTCGTTTGATCGGTTTTAATTACAGTTTTT TTATTGTTAATTTCTCTTCCTGTTCTTGCAGGAGCAATTACAATATTATTAATAGATCGA AACTTTAATAGATCTTTCTATGACCCTCTGGGGGGAGGAGACC JSTR00056_0103 TGTTTTATATTTTATCTTTGGTGTCTGGAGTGGTTTAATTGGGACTTCTTTTAGTATTATT ATTCGTTCTGAACTTATAAATGTAGGACCATTTTTGGCAAATGACCATTTGTATAATGTT GTAGTTACTTCTCATGCGTTTATTATAATCTTTTTTATAACGATACCTTTAGTTATCGGT GGATTTGGGAATTGATTAATTCCTCTAATAATTGGTGCTCCTGACATAGCTTTTCCTCGT ATAAATAATTTAAGGTTTTGACTTCTTGTTCCTTCTCTAATTTTTATATTAATGAGAATA GTTATTAGTCTAGGATCAGGTACTGGTTGGACGGTGTACCCTCCCCTGTCAATAAGACT GACTCACAGGGGGCTTTCGGTTGATCTATTAATTTTTTCTTTACATATTGCAGGTGTGTC TTCAATTTTAGGGTCAGTAAATTTTATTGTTACCATTTTTAATATACGTGTTGTTGGTAT 
AAATTTTGAGTATCTAAGTTTATTTGTGTGATCTGTATTAATTACGGTGTTTTTATTACT TATTTCTCTTCCTGTTCTCGCAGGAGCGATCACTATGTTACTGATGGACCGAAATTTTAA TAGATCTTTCTATGATCCTTTAGGAGGAGGAGATCCTATTTTGTATCAACACTTATTT

JSTR00056_0104

TATATTATACTTTATTTTTGGTATTTGGAGTGGTCTCATCGGAACTTCATTCAGGATGTT AATTCGGTCTGAATTGATAAGGGTGGGCTCTTTTCTGATAAATGATCAATTATATAATG TTGTTGTCACTTCACATGCGTTCATTATGATTTTTTTTATAACCATACCTTTAGTAATTG GTGGATTCGGGAACTGGCTAGTACCTCTTATAATCGGTGCGCCCGATATAGCGTTTCCT CGAATAAATAATCTTAGGTTTTGACTATTAATCCCCTCCTTAATATTTATAATAACTAGA ATGTTGGTGGGAAGCGGAGCTGGGACGGGGTGAACCGTTTATCCGCCCCTCTCATTGG GGTTAGCTCATAGTGGGTCTTCAGTTGATCTGCTGATCCTGGCCTTACATGTCGCAGGC ATCTCATCTATCTTAGGGTCTGTTAATTTTATTGTTACTATTTTCAACATGCGCGTACTG GGTATAAGATTTGAGTATGTTAGCTTGTTTGTTTGGTCTGTTTTAATCACTGTGTTTTTG TTGCTTATCTCTCTGCCCGTTTTGGCGGGGGCAATTACTATGCTACTGATGGATCGAAA TTTCAATAGGTCATTTTATGACCCCTTGGGGGGAGGGGATCCCGTTCTATACCAACATC TATTT

JSTR00056_0105

TGTTTTATATTTTATCTTTGGTGTCTGGAGTGGTTTAATTGGGACTTCTTTTAGTATTATT ATTCGTTCTGAACTTATAAATGTAGGACCATTTTTGGCAAATGACCATTTGTATAATGTT GTAGTTACTTCTCATGCGTTTATTATAATCTTTTTTATAACGATACCTTTAGTTATCGGT GGATTTGGGAATTGATTAATTCCTCTAATAATTGGTGCTCCTGACATAGCTTTTCCTCGT ATAAATAATTTAAGGTTTTGACTTCTTGTTCCTTCTCTAATTTTTATATTAATGAGAATA GTTATTAGTCTAGGATCAGGTACTGGTTGGACGGTGTACCCTCCCCTGTCAATAAGACT GACTCACAGGGGGCTTTCGGTTGATCTATTAATTTTTTCTTTACATATTGCAGGTGTGTC TTCAATTTTAGGGTCAGTAAATTTTATTGTTACCATTTTTAATATACGTGTTGTTGGTAT AAATTTTGAGTATCTAAGTTTATTTGTGTGATCTGTATTAATTACGGTGTTTTTATTACT TATTTCTCTTCCTGTTCTCGCAGGAGCGATCACTATGTTACTGATGGACCGAAATTTTAA TAGATCTTTCTATGATCCTTTAGGAGGAGGAGATCCTATTTTGTATCAACACTTATTT

Figure 1 : Séquences nucléotiques de 8 adultes de Bemisia présents dans nos échantillons.

JSTR00056_0201

AATTTTATATTTTATTTTTGGGATTTGAAGAGGATTTATTGGAGCTTCCATAAGATTATT AATTCGTATAGAATTAAGAATGTTAGGTAGCTATTTAAATAATGATCAAATATATAATA CTTTAGTTACTTCTCATGCTTTTATTATAATTTTTTTCATAACAATACCTTTAGTTATTGG TGGGTTTGGTAATTGGTTAGTACCTTTAATAATTGGAGCTCCTGATATAGCTTTTCCTCG TATGAATAATTTAAGATTCTGATTATTAATTCCTTCATTAATTTTTTTAATTTTAAGAAT GTTAATTTCTAGAGGAGGTGGCACAGGTTGAACAGTTTACCCTCCTTTATCTACAATTA TATTTCATAGAGGTTGTTCAGTTGACTTAATAATTTTTTCTTTACATTTAGCTGGGATTT CTTCTATTTTGGGTTCAATTAATTTTATTACTACAATTGTTAATATGCGTTTATTTGGGA TAAAATTGGAAAATATATCTTTATTTGTTTGGTCAGTATTTATTACTGTTTTTTTATTATT ATTATCTTTACCTGTTTTAGCAGGAGCTATTACTATGTTATTATTAGATCGAAATTTTAA TAGAACATTTTTTGATCCTATGGGAGGGGGTGACCCTATTTTATACCAACATTTATTT JSTR00056_0202 AATTTTATATTTTATTTTTGGGATTTGAAGAGGATTTATTGGAGCTTCCATAAGATTATT AATTCGTATAGAATTAAGAATGTTAGGTAGCTATTTAAATAATGATCAAATATATAATA CTTTAGTTACTTCTCATGCTTTTATTATAATTTTTTTCATAACAATACCTTTAGTTATTGG 
TGGGTTTGGTAATTGGTTAGTACCTTTAATAATTGGAGCTCCTGATATAGCTTTTCCTCG TATGAATAATTTAAGATTCTGATTATTAATTCCTTCATTAATTTTTTTAATTTTAAGAAT GTTAATTTCTAGAGGAGGTGGCACAGGTTGAACAGTTTACCCTCCTTTATCTACAATTA TATTTCATAGAGGTTGTTCAGTTGACTTAATAATTTTTTCTTTACATTTAGCTGGGATTT CTTCTATTTTGGGTTCAATTAATTTTATTACTACAATTGTTAATATGCGTTTATTTGGGA TAAAATTGGAAAATATATCTTTATTTGTTTGGTCAGTATTTATTACTGTTTTTTTATTATT ATTATCTTTACCTGTTTTAGCAGGAGCTATTACTATGTTATTATTAGATCGAAATTTTAA TAGAACATTTTTTGATCCTATGGGAGGGGGTGACCCTATTTTATACCAACATTTATTT JSTR00056_0203 AATTTTATATTTTATTTTTGGGATTTGAAGAGGATTTATTGGAGCTTCCATAAGATTATT AATTCGTATAGAATTAAGAATGTTAGGTAGCTATTTAAATAATGATCAAATATATAATA CTTTAGTTACTTCTCATGCTTTTATTATAATTTTTTTCATAACAATACCTTTAGTTATTGG TGGGTTTGGTAATTGGTTAGTACCTTTAATAATTGGAGCTCCTGATATAGCTTTTCCTCG TATGAATAATTTAAGATTCTGATTATTAATTCCTTCATTAATTTTTTTAATTTTAAGAAT GTTAATTTCTAGAGGAGGTGGCACAGGTTGAACAGTTTACCCTCCTTTATCTACAATTA TATTTCATAGAGGTTGTTCAGTTGACTTAATAATTTTTTCTTTACATTTAGCTGGGATTT CTTCTATTTTGGGTTCAATTAATTTTATTACTACAATTGTTAATATGCGTTTATTTGGGA TAAAATTGGAAAATATATCTTTATTTGTTTGGTCAGTATTTATTACTGTTTTTTTATTATT ATTATCTTTACCTGTTTTAGCAGGAGCTATTACTATGTTATTATTAGATCGAAATTTTAA TAGAACATTTTTTGATCCTATGGGAGGGGGTGACCCTATTTTATACCAACATTTATTT JSTR00056_0204 AATTTTATATTTTATTTTTGGGATTTGAAGAGGATTTATTGGAGCTTCCATAAGATTATT AATTCGTATAGAATTAAGAATGTTAGGTAGCTATTTAAATAATGATCAAATATATAATA CTTTAGTTACTTCTCATGCTTTTATTATAATTTTTTTCATAACAATACCTTTAGTTATTGG TGGGTTTGGTAATTGGTTAGTACCTTTAATAATTGGAGCTCCTGATATAGCTTTTCCTCG TATGAATAATTTAAGATTCTGATTATTAATTCCTTCATTAATTTTTTTAATTTTAAGAAT GTTAATTTCTAGAGGAGGTGGCACAGGTTGAACAGTTTACCCTCCTTTATCTACAATTA TATTTCATAGAGGTTGTTCAGTTGACTTAATAATTTTTTCTTTACATTTAGCTGGGATTT CTTCTATTTTGGGTTCAATTAATTTTATTACTACAATTGTTAATATGCGTTTATTTGGGA TAAAATTGGAAAATATATCTTTATTTGTTTGGTCAGTATTTATTACTGTTTTTTTATTATT ATTATCTTTACCTGTTTTAGCAGGAGCTATTACTATGTTATTATTAGATCGAAATTTTAA TAGAACATTTTTTGATCCTATGGGAGGGGGTGACCCTATTTTATACCAACATTTATTT

JSTR00056_0205

AATTTTATATTTTATTTTTGGGATTTGAAGAGGATTTATTGGAGCTTCCATAAGATTATT AATTCGTATAGAATTAAGAATGTTAGGTAGCTATTTAAATAATGATCAAATATATAATA CTTTAGTTACTTCTCATGCTTTTATTATAATTTTTTTCATAACAATACCTTTAGTTATTGG TGGGTTTGGTAATTGGTTAGTACCTTTAATAATTGGAGCTCCTGATATAGCTTTTCCTCG TATGAATAATTTAAGATTCTGATTATTAATTCCTTCATTAATTTTTTTAATTTTAAGAAT GTTAATTTCTAGAGGAGGTGGCACAGGTTGAACAGTTTACCCTCCTTTATCTACAATTA TATTTCATAGAGGTTGTTCAGTTGACTTAATAATTTTTTCTTTACATTTAGCTGGGATTT CTTCTATTTTGGGTTCAATTAATTTTATTACTACAATTGTTAATATGCGTTTATTTGGGA TAAAATTGGAAAATATATCTTTATTTGTTTGGTCAGTATTTATTACTGTTTTTTTATTATT ATTATCTTTACCTGTTTTAGCAGGAGCTATTACTATGTTATTATTAGATCGAAATTTTAA TAGAACATTTTTTGATCCTATGGGAGGGGGTGACCCTATTTTATACCAACATTTATTT

Figure 2 : Séquences nucléotiques de 5 adultes d'Aleurodicus présents dans nos échantillons. 


\section{DISCUSSION}

A. dispersus est une espèce néotropicale en pleine expansion au niveau mondial. Elle a été recensée dans les pays africains suivants : Bénin, Cameroun, Congo, Ghana et Nigeria par Evans (2007) et au Kenya par Mware (2010). A notre connaissance, c'est la première fois que cette espèce est signalée du Sénégal. C'est une espèce très polyphage connue pour attaquer notamment des arbustes d'ornement. Elle a été signalée sur plusieurs espèces de Solanaceae, notamment des Solanum et Capsicum. Il n'est pas étonnant de la trouver sur tomate, bien qu'il ne s'agisse vraisemblablement pas de sa plante hôte de prédilection. Selon Jones (2003) A. dispersus n'est pas connu pour transmettre des viroses même si elle est suspectée de transmettre le Cassava Brown Streak virus disease (Mware, 2010). Il est aussi probable qu'il demeure un ravageur secondaire sur tomate mais pourrait causer bien plus de dégâts à de nombreuses autres espèces cultivées au Sénégal.

Deux espèces différentes de Bemisia ont été identifiées sur les tomates cultivées dans le site. B. tabaci avec certitude et une espèce probablement proche de $B$. afer. Concernant cette deuxième espèce c'est à notre connaissance la première fois qu'elle est signalée au Sénégal.

La recrudescence de Bemisia spp, en rapport avec la virose de la tomate pourrait être corrélée à l'existence de souches de plus en plus virulentes ou agressives. En effet dans certaines localités, les mouches blanches sont passées, en dix ans, de ravageurs secondaires à ravageurs principaux agricoles, avec des conséquences économiques importantes sur les productions de tomates (Traboulsi, 1994).

Il a été observé essentiellement des adultes sur tomate. Les adultes des complexes $B$. tabaci et $B$. afer sont quasiment identiques morphologiquement (Malumphy et al., 2009). La découverte de deux espèces en mélange pose le problème de la transmission du TYLC. $B$. tabaci est un vecteur bien connu de ce virus par contre, même si B. afer est suspecté de transmettre le Cassava Brown Streak virus disease (Jones, 2003), son implication dans la transmission d'autres virus est peu documentée. Cette espèce est par contre considérée comme un ravageur important des cultures en Afrique sub-saharienne (Malumphy, 2003). Cette dominante dans les échantillons prélevés et majoritairement identifiés comme Bemisia spp serait certainement responsable de la transmission du TYLCV au Sénégal.

\section{Conclusion}

L'étude sur les espèces d'aleurodes et le $T Y L C$, virose de la tomate, dans la zone des Niayes au Sénégal a mis en évidence deux espèces différentes de Bemisia : B. tabaci et $B$. afer (signalée pour la première fois au Sénégal) et Aleurodicus dispersus. Cependant, la recrudescence de Bemisia spp, en rapport avec la virose de la tomate, pourrait être corrélée à l'existence de souches de plus en plus virulentes ou agressives. La présence de A. dispersus dans nos échantillons qui semble être le vecteur de Cassava Brown Streak virus disease nous recommande de continuer les prospections pour savoir si cette espèce s'étend au Sénégal et fait des dégâts et laquelle des deux Bemisia domine.

\section{CONFLITS D'INTERETS}

Tous les quatre auteurs ont contribué à la révision de la dernière version de l'article et ont tous donné leur accord pour la publication. Aussi, nous tous déclarons sur l'honneur l'absence de tout conflit d'intérêt pour cet article.

\section{CONTRIBUTIONS DES AUTEURS}

Les quatre auteurs comprennent un phytopathologiste, acteur principal dans la collecte, l'analyse et la rédaction du premier draft; un écologiste sur l'analyse de la diversité spatiale et l'amélioration du document; un entomologiste pour l'analyse morphologique et la révision des différentes versions et un malherbologue pour sa contribution sur les plantes réservoirs dans la zone d'étude et la finalisation de cet article. 


\section{REMERCIEMENTS}

Nous remercions la collaboration du CBGP (Centre de Biologie pour la Gestion des Populations) de Montpellier, Campus International de Baillarguet, en particulier M. Jean Claude STREITO, pour les travaux de laboratoire qu'il a bien voulu nous réaliser avec professionnalisme et disponibilité.

\section{RÉFÉRENCES}

Bouchelta A, Boughdad A. et Blenzar A. 2005. Effets biocides des alcaloïdes, des saponines et des flavonoïdes extraits de Capsicum frutescens L. (Solanaceae) sur Bemisia tabaci (Gennadius). BASE, 9(4): 259-269.

Brown JK. 1994. Current status of Bemisia tabaci as a plant pest and virus vector in agroecosystems worldwide. FAO Plant Protection Bulletin, 42(1 - 2): 3 - 32.

Camara M. 2012. Contribution à l'étude de la maladie virale du jaunissement et de l'enroulement en cuillère des feuilles de tomate (Lycopersicon esculentum Mill.), causée par le Tomato Yellow Leaf Curl Virus (TYLCV), dans la zone maraîchère des Niayes de Dakar. Thèse de Doctorat d'Etat ès Sciences Naturelles. Faculté des Sciences et Techniques. Université Cheikh Anta Diop de Dakar. 141p.

Ciss I. 2004. Epidémiologie comparée du TYLCV chez les variétés sensibles et tolérantes de tomate (Lycopersicon esculentum Mill). Mémoire de fin d'études pour l'obtention du Diplôme d'Ingénieur des Travaux Agricoles. Ecole Nationale des Cadres Ruraux de Bambey. Sénégal, 43p.

De Barro JP, Shu-Sheng L, Boykin, Laura M, Dinsdale AB. 2011. Bemisia tabaci: A statement of Species Status. Annu. Rev. Entomol., 56:1-19.

Delatre R. 1973. Parasites et Maladies en Culture Cotonnière. Edit. Paris Division de Documentation de l'I.R.C.T : Paris, France ; 146p.

Diatta R. 1999. Dynamique des populations et efficacités d'aleurocides sur la mouche blanche du cotonnier Bemisia tabaci.
Mémoire de fin d'études pour l'obtention du diplôme d'ingénieur des travaux agricoles. ENCR de Bambey, Sénégal. $33 p$.

Evans GA. 2007. The whiteflies (Hemiptera: Aleyrodidae) of the world and their host plants and natural enemies. http://www.sel.barc.usda.gov:591/1WF/w hitefly_catalog.htm. Version070606, 11 June 2007. 708p.

Frohlich DR, Torres-Jerez I, Bedford ID, Markham PG, Brown JK. 1999. A phylogeographical analysis of the Bemisia tabaci species complex sed on mitochondrial DNA markers. Molecular Ecology, 8(10): 1683-1691.

Guimaraës JM. 1996. The diagnostic value of the cement gland and other abdominal structures in aleyrodid taxonomy. EPPO Bull. OEPP., 26(2): 413-419.

Jones DR. 2003. Plant Viruses transmitted by whiteflies. European Journal of Plant Pathology, 109: 195-219.

Malumphy CH, Walsh K, Suarez MB, Collins DW, Boonham N. 2009. Morphological and molecular identification of all developmental stages of four whitefly species (Hemiptera: Aleyrodidae) commonly intercepted in quarantine. Zootaxa, 2118: 1-29.

Malumphy CH. 2003. The status of Bemisia afer (Priesner \& Hosny) in Britain (Homoptera: Aleyrodidae). Entomologist's Gazette, 54: 191-196.

Martin JH. 2003. Whiteflies (Hemiptera: Aleyrodidea) their systematic history and the resulting problems of conventional taxonomy, with special reference to descriptions of Aleyrodes proletella (Linnaeus, 1758) and Bemisia tabaci (Gennadius, 1889). Entomologist Gazette, 54: 125-136.

Messiaen CM, Blancard D, Rouxel F, Lafon R. 1991. Les Maladies des Plantes Maraîchères ( $3^{\text {ème }}$ édn). INRA Publications : Paris, France; 547p.

Mware B, Olubayo F, Narla R, Songa J, Amata R, Kyamanywa S, Ateka EM. 2010. First Record of Spiraling Whitefly 
in Coastal Kenya: Emergence, Host Range, Distribution and Association with Cassava Brown Streak Virus Disease. International Journal of Agriculture \& Biology, 12(3): 411-415.

Reynaud P. 2000. L'aleurode Bemisia tabaci en France. Situation actuelle et possibilités de développement. PHYTOMA-La Défense des Végétaux 527, 18-21. Schulten GGM. 1997. Overview of the whitefly-virus problem: objectives of the workshop. FAO Plant Prod. Prot. Paper. 143, p. 7-10.

Secker AE, Bedford ID, Markham PG, Williams MEC. 1998: Squash, a reliable field indicator for the presence of the $\mathrm{B}$ biotype of tobacco whitefly, Bemisia tabaci - Brighton Crop Protection Conference. Pests \& Diseases, 3: 837842.

Streito JC. 2004. Découverte en France de Crenidorsum aroidephagus Martin \& Aguiar, 2001, description de la femelle, caractères permettant de la différencier d'autres aleurodes d'intérêt agronomique (Hemiptera, Aleyrodidae). Nouvelle Revue d'Entomologie, 21(4): 375-382.

Traboulsi R. 1994. Bemisia tabaci: A report on its pest status with particular reference to the Near East. FAO Plant Prot. Bull, 42(1-2): 33-58. 